\title{
Articles
}

\section{CLUSTERING MULTIPLE SCLEROSIS SUBGROUPS WITH MULTIFRACTAL METHODS AND SELF-ORGANIZING MAP ALGORITHM}

\author{
YELIZ KARACA* and CARLO CATTANI ${ }^{\dagger}$ \\ Engineering School (DEIM) \\ Tuscia University, 01100 Viterbo, Italy \\ *yeliz.karaca@ieee.org \\ ${ }^{\dagger}$ cattani@unitus.it
}

Received February 23, 2017

Revised April 5, 2017

Accepted April 10, 2017

Published June 9, 2017

\begin{abstract}
Magnetic resonance imaging (MRI) is the most sensitive method to detect chronic nervous system diseases such as multiple sclerosis (MS). In this paper, Brownian motion Hölder regularity functions (polynomial, periodic (sine), exponential) for 2D image, such as multifractal methods were applied to MR brain images, aiming to easily identify distressed regions, in MS patients. With these regions, we have proposed an MS classification based on the multifractal method by using the Self-Organizing Map (SOM) algorithm. Thus, we obtained a cluster analysis by identifying pixels from distressed regions in MR images through multifractal methods and by diagnosing subgroups of MS patients through artificial neural networks.
\end{abstract}

Keywords: Multifractals; Hölder Exponent; MRI; MS Subgroups; SOM.

\footnotetext{
${ }^{*}$ Corresponding author.

This is an Open Access article published by World Scientific Publishing Company. It is distributed under the terms of the Creative Commons Attribution 4.0 (CC-BY) License. Further distribution of this work is permitted, provided the original work is properly cited.
} 


\section{INTRODUCTION}

Fractal theory was developed taking into consideration the analysis of irregular objects. Abundant natural phenomena as physics, biology as well as medicine have been revealed to show a fractal behavior.

Multiple Sclerosis (MS) is a central nervous system and chronic disease created by the immune system weakening myelin, which is the protein surrounding neural tissue. ${ }^{12}$ The clinical course types are defined into such MS subgroup titles as Benign MS, Relapsing Remitting MS (RRMS), Secondary Progressive MS (SPMS), Progressive Relapsing MS (PRMS), Primary Progressive MS (PPMS) (for some more information on MS and subgroups, see e.g. Refs. 1-4). Magnetic Resonance Imaging (MRI) can reveal inflammatory tissue regions in the central nervous system, thus being one of the most important tools for MS diagnosis.

In recent years, there has been an increasing interest for the fractal analysis of images in biomedical applications. Regarding the relevant topics, in the following studies, Cattani et al., 5 Trujillo et al. ${ }^{6} \mathrm{Yu}$ and Qi, ${ }^{7}$ Phothisonothai and Nakagawa emphasized the importance of fractal methods for data analysis in the field of medicine. Various studies have been done on the analysis of the medical image data and on MRI data. Zook and Iftekharuddin,,$\frac{9}{9}$ Esteban et al., $\frac{100}{10}$ Akar et al.,11 Abirami and Karthik $^{12}$ and Lahmiri and Boukadoum ${ }^{13}$ carried out the detection of tumorous regions (white matter and cerebrospinal fluid) on MRI data through multifractal algorithms.

Our paper deals with the multifractal 2D pixel analysis of MRI obtained from some MS patients and healthy individuals. Brownian Motion and Hölder exponent functions (polynomial, periodic and exponential) are computed for the MRI regularity. We obtained significant information on the disrupted regions (lesion diameters and lesion diameter sizes) of the brain. These pixels are trained by using the self-organizing maps (SOM) algorithm. In particular, 2D pixels, obtained from the multifractal analysis, are clustered by the SOM algorithm. The data obtained from Hölder polynomial, periodic and exponential functions was trained by SOM algorithm. Hölder periodical function had a better clustering of MS subgroups and healthy individuals compared to other functions.

Compared to recent papers on MRI of similar data images, 81013 our approach is more general and complete since we have taken data from a larger sample (139 individuals), from different regions of the brain, in three different years to analyze some time dependence as well. In particular, the multifractal analysis of MRI data (from MS) has been applied for the first time for the three regions of the brain, having obtained more significant information for MS diagnosis.

\section{DATA AND METHOD}

\subsection{Patient Details}

In this study, MRI data of 120 MS patients and 19 healthy individuals, aged 20-65, distributed with respect to gender and age according to Table 1, were collected at the Hacettepe University Neurology and Radiology Department, Primer Medical Imaging Center (Ankara, Turkey). The images were obtained by using an MRI scanner of 1.5 Tesla. 14

MRI were taken from 120 MS subgroup patients (76 RRMS, 38 SPMS, 6 PPMS) and 19 healthy (Not-MS) individuals in three different nonconsecutive years (with a minimum of three years between the first and second MRI and a maximum of eight years between the second and third ones).

\subsection{Magnetic Resonance Imaging}

MRI is an important medical practice to diagnose neurological diseases like MS. 1415 MRI are used for displaying soft tissues in the brain and the spinal cord. An MRI can reveal inflammatory or damaged tissue in regions of the central nervous system. In this analysis, we have considered the MRI data taken in three regions of the brain (upper brainstem, corpus callosum-periventricular region, upper cervical region). In particular, MRI from 120 MS patients and 19 healthy individuals have been analyzed, in three different nonconsecutive years, with patients belonging to the following subgroups: 76 RRMS, 38 SPMS and 6 PPMS patients.

\subsection{Methods}

Our aim is to classify the MS disease through the multifractal analysis of the MRI. Our method is

Table 1 MS Patient Age.

\begin{tabular}{lrrrrrrr}
\hline Age & $\mathbf{1 8 - 2 5}$ & $\mathbf{2 6 - 3 0}$ & $\mathbf{3 1 - 3 5}$ & $\mathbf{3 6 - 4 0}$ & $\mathbf{4 1 - 4 5}$ & $\mathbf{4 6 - 5 0}$ & $\mathbf{5 1 - 6 5}$ \\
\hline Female & 10 & 22 & 17 & 16 & 9 & 8 & 6 \\
Male & 6 & 11 & 4 & 6 & 4 & 0 & 1 \\
\hline
\end{tabular}


based on the following steps:

(a) Multifractal analysis was applied to MRI.

(b) Brownian motion Hölder regularity (polynomial, periodic (sine) and exponential) for $2 \mathrm{D}$ pixel analysis were applied to MRI so that we could identify those significant disrupted regions on the MRI. Lesions were classified according to lesion diameters and lesion sizes.

(c) The wounded regions and lesions were finally clustered by using the Kohonen SOM algorithm.

Computations and figures were obtained by MATLAB and FracLab environment.

\subsubsection{Two-dimensional fractional Brownian motion}

The fractional Brownian motion $(f B m)$ function $B_{H}(t)$ is (see e.g. Ref. 16).

$$
\begin{aligned}
\operatorname{cov}\{ & \left.B_{H}(S), B_{H}(t)\right\} \\
& =\frac{1}{2}\left\{|S|^{2 H}+|t|^{2 H}-|S-t|^{2 H}\right\} .
\end{aligned}
$$

Let $B_{H}(t)$ the $f B m$ defined by the stochastic integral be

$$
\begin{aligned}
B_{H}(t)= & \frac{1}{\Gamma(H+(1 / 2))} \times\left\{\int _ { - \infty } ^ { 0 } \left[(t-S)^{H-\left(\frac{1}{2}\right)}\right.\right. \\
& \left.\left.-(-S)(t-S)^{H-\left(\frac{1}{2}\right)}\right]\right\} d W(S) \\
& +\int_{-\infty}^{0}\left[(t-S)^{H-\left(\frac{1}{2}\right)} d W(S)\right]
\end{aligned}
$$

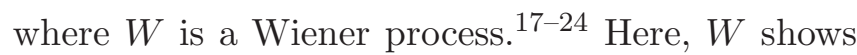
the ordinary Brownian motion. In addition, integration is taken in the mean square form. This kind of a natural extension of $f B m$ leads to some sense of a "loss" of properties in that the increments of $f B m$ in fact are nonstationary and the process is not self-similar anymore. It is known that irregularities and singularities are the most prominent ones and informative components of a pixel.

$H$ being the Hurst exponent, $H=\alpha / 2$ and $\alpha \in$ $(0,1)$ is the fractal dimension.17/21

$H$ assumes two major roles. The first one is that it makes the determination of the roughness of the sample path besides the long range dependence properties pertaining to the process. Most of the time, the roughness of the path is based on the location. It may also be necessary to take the multiple regimes into consideration. As for the second role, it can be said that this role can be realized by replacing the $H$ by a Hölder function $H(t)$ as can be seen below 23 :

$$
0<H(t)<1, \quad \forall t .
$$

This kind of a generalization gives the way to multifractional Brownian motion $(m f B m)$ term. In such a case, the integral expression resembles the one pertaining to the $f B m$, but the case of $H$ being replaced by the corresponding Hölder function is not applicable. The second integral is with an upper limit of $t$ and the integration is taken in the mean square sense. ${ }^{20}$ In addition, for $m f B m$, increments are not stationary anymore and the process is not self-similar anymore either. Two-dimensional Fractional Brownian Motion (2D-fBm) algorithms form time series of spatial coordinates.20 25

\subsubsection{Pointwise Hölder exponent}

Hölder function of exponent $\beta: \operatorname{Let}\left(X, d_{x}\right)$ and $\left(Y, d_{y}\right)$ be two metric spaces. A function $f: X \rightarrow Y$ is named as a Hölder function of exponent $\beta>0$, if for each $x, y \in X$ such that $d_{X}(x, y)<1$, it is 22 :

$$
d_{Y}(f(x), f(y)) \leq C \cdot d_{x}(x, y)^{\beta},
$$

$f$ is a function from an interval $D \subseteq R$ into $R$. The function $f$ is Hölder continuous if there are positive constants $C$ and $\beta$ with

$$
|f(x)-f(y)| \leq C|x-y|^{\beta}, \quad x, y \in D .
$$

The constant $\beta$ is called the Hölder exponent. Hölder continuity enables the measurement of the rate of convergence. If $f$ is Hölder continuous with exponent $\beta$, then $f(x)-f(y) \rightarrow 0$ as $x \rightarrow y$ at least in the same fastness as $|x-y|^{\beta} \rightarrow 0 . \underline{26} \underline{28}$

\subsubsection{Hölder regularity}

Singular and irregular structures are some of the most useful information within a signal analogously irregular patterns in images are used for recognition tasks. [6] Hölder regularity is a manner characterizing these singular structures in a precise way 13122 It is possible to quantify the regularity of a signal at each point by the pointwise Hölder exponent as the image stated below.

It is important to be able to obtain significant pixel value from an image. Hölder regularity characterizes significant singularities. ${ }^{6] 29}$ In Eq. (6), each point in an image pixel is defined according to Hölder exponent as provided above. 
Let $f: R \rightarrow R, S \in R^{+*} \backslash N$ and $x_{0} \in R . f \in$ $C^{S}\left(x_{0}\right)$ if and only if $\exists \eta \in R^{+*}$ and polynomial degree $P$ of degree $<S$ and a constant $C$ such that $\underline{6}$

$$
\left(\forall x \in B\left(x_{0}, \eta\right)\left|f(x)-P\left(x-x_{0}\right)\right| \leq C\left|x-x_{0}\right|^{s}\right)
$$

in which $B\left(x_{0}, \eta\right)$ is the local neighborhood around $x_{0}$ with a radius $\eta$. The pointwise Hölder exponent of $f$ at $x_{0}$ is as $\alpha_{p}\left(x_{0}\right)=\beta^{\left(\alpha_{p} r+p\right)}\left(x_{0}\right)=\sup _{s}\{f \in$ $\left.C^{s}\left(x_{0}\right)\right\}$.

The Hölder exponent of function $f(t)$ at point $t$ is the $\sup \left(\alpha_{p}\right) \in[0,1]$, for which a constant $C$ exists such that $\forall t^{\prime}$ in a neighborhood of $t$,

$$
\left|f(t)-f\left(t^{\prime}\right)\right| \leq \mathrm{C}\left|t-t^{\prime}\right|^{\alpha_{p}} .
$$

As for pixel oscillations, a function $f(t)$ is Hölder with exponent $\alpha_{p} \in[0,1],\left(\alpha_{p}=\beta^{\left(\alpha_{p} r+p\right)}\right)$ at $t$ if $\exists c \forall \tau$ such that $\operatorname{osc}_{\tau}(t) c \tau^{\alpha_{p}}$, with the following:

$$
\operatorname{osc}_{\tau}(t)=\sup _{t^{\prime}, t^{\prime \prime} \in|t-\tau, t+\tau|}\left|f\left(t^{\prime}\right)-f\left(t^{\prime \prime}\right)\right| .
$$

Now, if $t=x_{0}$ and $t^{\prime}=x_{0}+h$ in Eq. (9) can also be written that

$$
\beta^{\left(\alpha_{p} r+p\right)}=\lim \inf _{h \rightarrow 0} \frac{\log \left|f\left(x_{0}+h\right)-f\left(x_{0}\right)\right|}{\log |h|} .
$$

For this reason, it is concerned with finding an $\alpha_{p}$ that fulfills Eqs. (8) and (91). To simplify this process, we can set $\tau=\beta^{r}$. Then, we can write $\operatorname{osc}_{\tau} \approx c \tau_{p}^{\alpha}=\beta\left(\alpha_{p} r+b\right)$, which is equivalent to as Eq. (10).

$$
\log _{\beta}\left(\operatorname{osc}_{\tau}\right) \approx\left(\alpha_{p} r+b\right) \approx\left(\beta^{\left(\alpha_{p} r+p\right)} r+p\right) .
$$

Hence, an estimation of the regularity can be built at each point by computing the slope of the regression between the logarithm of the oscillations $\operatorname{osc}_{\tau}$ and the logarithm of the dimension of the neighborhood at which the oscillations $\tau$ are calculated. Here, in this study, least squares regression has been used for the computation of the slope with $\beta=2$ and $r=1,2,3,4$. In addition, it is preferable not to use all sizes of neighborhoods between two values $\tau_{\min }$ and $\tau_{\max }$. In this study, point $x_{0}$ has been calculated only on intervals of the form $\left[x_{0}-\tau_{r}: x_{0}+\tau_{r}\right]$. For a $2 \mathrm{D}$ image pixel, $x_{0}$ defines a point in $2 \mathrm{D}$ image space and $\tau_{r}$ a radius of a circle centered at $x_{0}$, such that $d\left(t^{\prime}, t\right) \leq \tau_{r}$ : and $d\left(t^{\prime \prime}, t\right) \leq \tau_{r}$, in which $d(a, b)$ is the Euclidean distance between a and $b ! 29$

In the present study, Hölder regularity functions (polynomial, periodic, exponential functions) are applied to MRI that belongs to MS subgroups and healthy individuals. The functions of $H_{1}, H_{2}$ and $H_{3}$ are obtained by selecting the most significant pixel values (for $H$ function between 0 and 1 , it is well understood that singular and irregular structures contain the most prominent, and most useful, information within a pixel) by applying them to the MRI (lesion number, lesion diameter). Three different functions were applied on FracLab (https://project.inria.fr/fraclab/) program to point coordinates $(x, y)$, where pixels obtained from MRI are located.

- Polynomial: $H_{1}(x, y)=0.1+0.8 x y$.

- Periodic (Sine): $H_{2}(x, y)=0.5$

$$
\text { - Exponential: } H_{3}(x, y)=0.3+\frac{0.3}{1+e^{-100(x-y)}} \text {. }
$$

Pixel values of $H_{1}, H_{2}$ and $H_{3}$ functions have been clustered to the Kohonen SOM algorithm. As a result, the most accurate clustering has been achieved for MS subgroups and healthy individuals.

\subsubsection{Kohonen SOM algorithm}

Kohonen $\mathrm{SOM}^{30}$ is a special algorithm, with competitive learning rules, in the theory of artificial neural network. A common feature in all competitive learning rules, such as the Kohonen algorithm, 31 is that a competition strategy is applied to all or a part of processing elements. As a result of the competition, the weights of the winning processing element are continuously updated. In the Kohonen algorithm, there exists a single output layer of topologically-related neurons in addition to the input layer. As can be seen in Fig. 1, each input is connected to each neuron in the output layer.31 33 The algorithm first starts to run with random weights. Then, choosing any arbitrary input, we will search for the neuron with least Euclid distance to this input vector (It is well understood that singular and irregular structures contain the most prominent, and most useful, information within a pixel). We update all connections to this neuron. The connection input weights of the neuron to be clustered is updated in a way that comes close to the input vector (as can be seen in Figs. 10 and 1b). Together with such winning neuron, weights of the neurons, in its topological neighborhood, are also selected.

In an algorithm with $N$ number of processing elements (neurons) $i(i=1, \ldots, N)$ and $n$ inputs (from polynomial, exponential, periodic Hölder function) $x_{j}(j=1, \ldots, n)$, the weight vector is defined as $w_{i}$, 


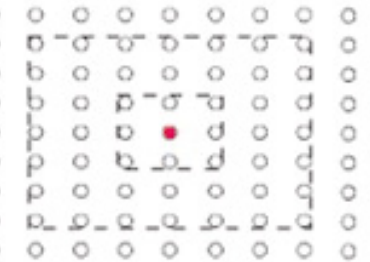

(a) Square

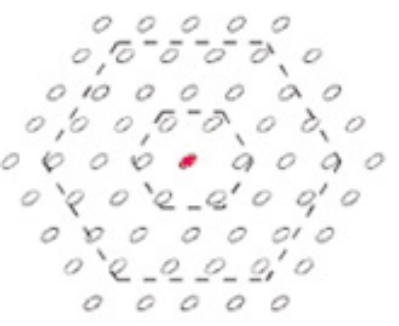

(b) Hexagon

Fig. 1 2D Kohonen algorithm and topological neighborhood relationship.

so that $w_{i j}$ represents the weight between the input $x_{j}$ and the neuron $i$. There follows that the intensity of the Kohonen processing element is the vector

$$
I_{i}=D\left(w_{i}, x\right), \quad\left(i=1, \ldots, N ; x=\left(x_{1}, \ldots, x_{n}\right)\right)
$$

where $D(u, \nu)$ shows the distance function. For example, if we take the Euclidian distance:

$$
D(u, \nu)=|u-\nu|=\left[(u-\nu)^{2}\right]^{1 / 2}
$$

with $(N=3, n=2)$, it is $w_{1}=\left(w_{11}, w_{12}\right), w_{2}=$ $\left(w_{21}, w_{22}\right), w_{3}=\left(w_{31}, w_{32}\right), x=\left(x_{1}, x_{2}\right)$ so that $I_{1}=D\left(w_{1}, x\right)=\left|w_{1}-x\right|=\left[\left(w_{1}-x\right)^{2}\right]^{1 / 2}=$ $\left[\left(w_{11}-x_{1}\right)^{2}+\left(w_{1}-x_{2}\right)^{2}\right]^{1 / 2}, \ldots$

We compute the input density of each neuron and we find the neuron with smallest input density, its output sign is taken as $Z_{i}=1 . \underline{31}$

The $w_{i}$ which is close to $x$ is found out as a result of competition. According to this explanation, we assume, as learning rule, the Kohonen learning rule:

$$
w_{i}^{\text {new }}=w_{i}^{\text {old }}+l\left(x-w_{i}^{\text {old }}\right) Z_{i},
$$

where $x$ is the input vector and $l$ is a constant generally ranging from 0 to 1 . If the winning neurons are defined as $Z_{i}=1$ and the losing ones as $Z_{i}=0$, there follows from Eq. (13).

$$
\begin{aligned}
& w_{i}^{\text {new }}=(1-l) w_{i}^{\text {old }}+l x,\{\text { winner }\}, \\
& w_{i}^{\text {new }}=w_{i}^{\text {old }},\{\text { loser }\} .
\end{aligned}
$$

The weight of the winner is continuously updated. In the clustering analysis of the input neuron, the weight of the neuron that wins the race so as to resemble the input neuron more is updated. In other words, there is an imitation for the purpose of being more similar to the probability density function of the input space. The Kohonen weights are dispersed in a form as if they would form a cloud around the $x$ input (as can be seen from Figs. П1a and पp).

This study aims at clustering the patients belonging to MS subgroups and healthy subjects in an accurate manner. The steps to achieve this aim can be seen as follows:

Step 1: Significant gray intensity (images of this sort are also known as black-and-white. It is a digital image where the value of each pixel is a single sample that merely carries intensity information, ranging from black with weakest intensity to white strongest) values of the MRI were calculated.

In this study, three different functions were applied on FracLab program to point coordinates $(x, y)$ where pixels obtained from MRI are located. First, the polynomial function $H_{1}(x, y)=0.1+$ $0.8 x y$ was applied, then the periodic one, $H_{2}(x, y)=$ $0.5+0.2(\sin (2 \pi x))\left(\cos \frac{3}{2} \pi y\right)$ as the second and the exponential one, $H_{3}(x, y)=0.3+\frac{0.3}{1+e^{-100(x-y)}}$ as the third. The matrix has a dimension of $128 \times 128$ and is obtained based on Hölder regularity functions. In MRI, each point signifies the pixel value. Our aim is to select the pixel values in line with the Hölder method. Such pixel values are regular and determine the disorder in the image belonging to the patients. For this study, the pixels with singular and irregular structures containing the most prominent, and most useful, information within a pixel are the significant values of ours. Significant values were selected based on the polynomial, exponential, periodic functions on Hölder regularity. Significant gray intensity displays the dense regions that have the same regularity based on Hölder regularity within the interval of $[0,1]$. The example of MRI provided in Fig. 3 makes the regions with lesion stand out in three different Hölder regularity functions. The significant pixel values selected from these functions were applied to the SOM algorithm. The clustering of MS subgroups and healthy subjects was made through the SOM algorithm. Table 4 provides information if the significant pixel values have determining attributes or not.

Step 2: Pixel values pertaining to MRI data were used. These were obtained from Hölder functions (polynomial, periodic and exponential). 


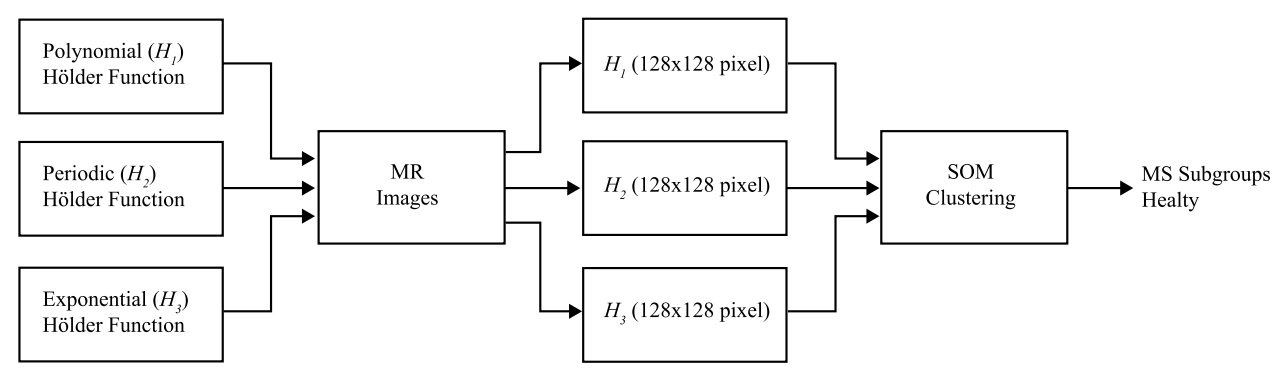

Fig. 2 Clustering the subgroups of MS through SOM algorithm by applying polynomial, periodic, exponential Hölder function to the MRI.

In this study, the SOM algorithm comprises $128 \times 128$ pixel inputs (gray intensity value) based on Hölder polynomial, periodic and exponential functions.

Step 3: Pixel values calculated were given as the input neuron to SOM algorithm. Thus, clustering analysis of MS subgroups was performed.

As can be seen in Fig. 2. polynomial, periodic, exponential Hölder function was applied to MRI. The most important pixel values $(128 \times 128 \mathrm{MRI}$ gray density) from the MRI based on each function were applied to the input layer of the SOM algorithm and they were trained in this way. For the SOM input layer, pixel values obtained from MRI were applied based on the $128 \times 128$ (new pixel inputs (gray intensity value)) Hölder functions. The output layer of the SOM algorithm refers to the MS subgroups regarding the relevant MS subgroups (RRMS, SPMS, PPMS) as well as the healthy subjects. As a result of the training procedure, MS subgroups were clustered.

\section{RESULTS AND DISCUSSION}

\subsection{Analysis of Hölder Regularity}

An $m f B m$ 2D synthesis analysis was carried out using the MRI data set for an MS subgroup and healthy individuals. The aim is to perform a cluster analysis by applying, through the SOM algorithm

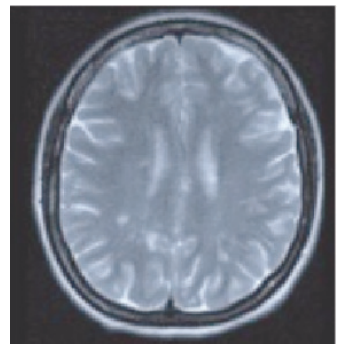

(a) Original MRI

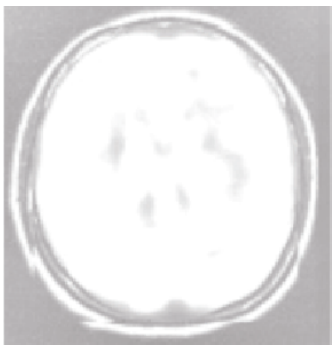

(b) Polynomial MRI $\left(H_{1}\right)$

method, those significant pixels obtained through applying the stochastic Brownian motion 2D pixel analysis to the MRI data set for three different functions.

In present study, three different functions were applied on FracLab program to point coordinates $(x, y)$ with pixels collected from MRI are located. First, the polynomial function $H_{1}(x, y)=0.1+$ $0.8 x y$ was applied, then the periodic one $H_{2}(x, y)=$ $0.5+0.2(\sin (2 \pi x))\left(\cos \frac{3}{2} \pi y\right)$ as the second and the exponential one $H_{3}(x, y)=0.3+\frac{0.3}{1+e^{-100(x-y)}}$ as the third. (The steps thereof were given in detail above.) The MRI in Fig. 3 belongs to one of the RRMS subgroup patients selected from the data we used in the analyses.

Figure 3 presents a visual representation of a type of output that this algorithm generates. This presents a sample image and the matching Hölder exponent function for each pixel. This method has proven to be superior ${ }^{8}$ in some cases to the wavelet leaders method ${ }^{25}$ and useful in applications of real-world. For this reason, we use the oscillations method for the comparisons with our evolved estimators.

In this study, the functions of $H_{1}, H_{2}$ and $H_{3}$ were obtained by selecting the most significant pixel values by applying them to the MRI (lesion number and lesion diameter). Significant pixel values of these three functions $H_{1}, H_{2}$ and $H_{3}$ have been

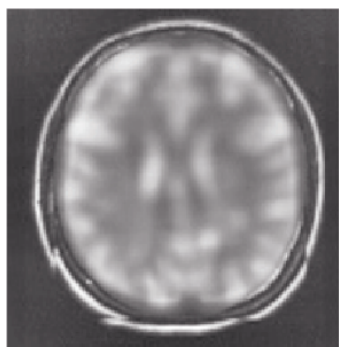

(c) Periodic MRI $\left(\mathrm{H}_{2}\right)$

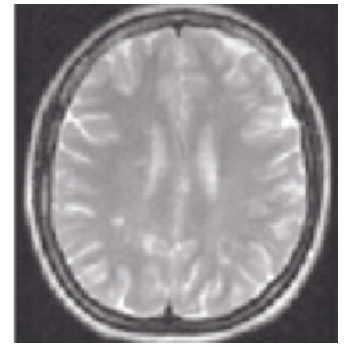

(d) Exponential MRI $\left(H_{3}\right)$

Fig. 3 A representative example of Hölder functions to MRI of an RRMS patient. 
applied to the Kohonen SOM algorithm in order to get the most accurate classification.

\subsection{Application of SOM}

Three different functions of the $\mathrm{mfBm}$ (polynomial, periodic (sine), exponential) were applied to MRI data taken in three different years from $120 \mathrm{MS}$ patients and 19 healthy individuals. Three different functions for $2 \mathrm{D}$ pixels were obtained as a result of the application. 2D pixels so obtained were clustered as RRMS, SPMS, PPMS, healthy (Not-MS) by the SOM algorithm. The highest SOM cluster accuracy in the multifractal function was obtained from periodic, exponential and polynomial analyses, respectively.

The common parameters used for the analysis of pixels through SOM algorithm are provided in Table 2. These parameters have yielded the best clustering accuracy for the current study. Because such parameters helped the best cluster analysis to be carried out in this study.

Here, 139 images for each of the prescribed regularity functions were generated. $H_{1}, H_{2}$ and $H_{3}$ all

Table 2 SOM Parameters in Experimental Analysis.

\begin{tabular}{lc}
\hline SOM Parameters & Description \\
\hline Topology function & Hexagonal layer \\
The distance function & Link distance \\
The ordering phase learning rate & 0.9 \\
Ordering phase steps & 1000 \\
The tuning phase learning rate & 0.02 \\
The neighborhood distance & 1.0 \\
\hline
\end{tabular}

images have the same size with $128 \times 128$ pixels. A total of 128 pixels were selected as the matrix size through Hölder polynomial, periodic, exponential functions. These pixel values of 128 were applied as the input neuron to the SOM algorithm for the clustering of MS subgroups and healthy subjects. The result of the obtained training analysis is presented in Fig. 4 .

In Figs. $4 a 4$ r, blue hexagons represent neurons. A red line connects neighbor neurons to one another. Colors in regions containing red lines show distances between neurons. Darker colors represent larger distances and lighter ones represents smaller distances. After application of the Hölder functions to MRI data, the SOM algorithm could group the MS subgroups and the healthy group into four individual clusters (RRMS, SPMS, PPMS, healthy).

In Table 3, the SOM topology is provided based on the result from SOM algorithm training processing according to the Hölder functions. A total of 128 distinguishing pixels were selected from MRI data for polynomial, periodic and exponential Hölder functions. Table 3 provides the number of individuals who can be clustered accurately by the SOM algorithm regarding the pixel values in the MRI of MS patients with subgroups and healthy individuals. Table 3 provides the clustering estimations of the MS subgroups and healthy individuals based on Hölder regularity functions. For example, the MRI of 12 individuals, who are known to have RRMS, were clustered accurately based on the pixel values selected from polynomial Hölder regularity function, which can be seen in Table 3a. According to Table 4, following the selection of distinguishing pixels, the periodic function provided a cluster rate

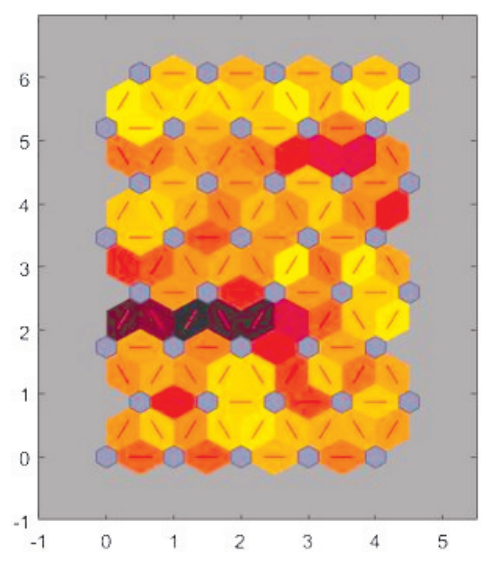

(a) Polynomial

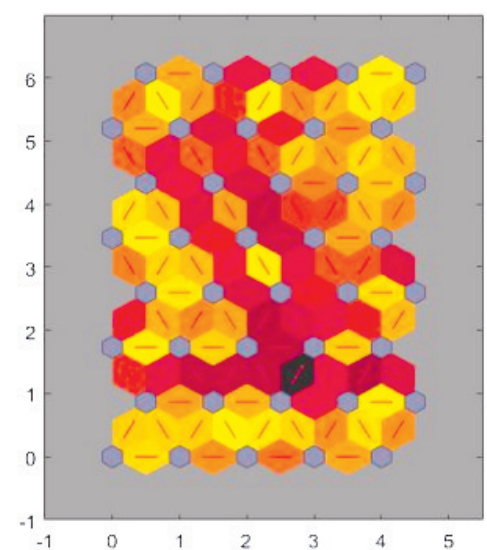

(b) Periodic

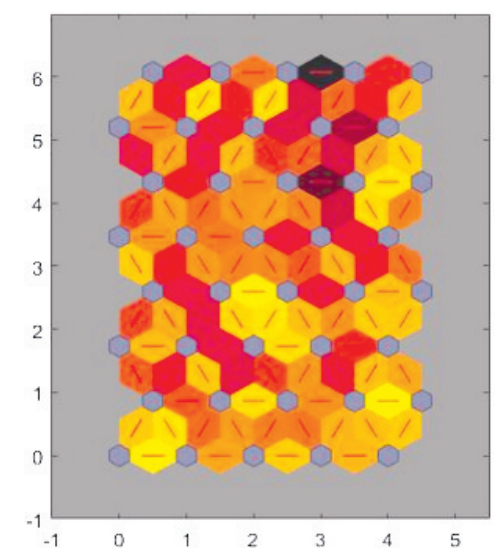

(c) Exponential

Fig. 4 SOM neighbor weight distance according to Hölder Functions. 
Table 3 According to Hölder Function SOM Topology for MRI.

\begin{tabular}{lrccc}
\hline & RRMS & SPMS & PPMS & Healthy \\
\hline \multicolumn{5}{c}{ (a) Polynomial } \\
RRMS & 12 & 9 & 0 & 0 \\
SPMS & 14 & 12 & 0 & 0 \\
PPMS & 3 & 17 & 11 & 0 \\
Healthy & 31 & 0 & 8 & 10 \\
\hline & \multicolumn{5}{c}{ (b) Periodic } & \\
RRMS & 15 & 6 & 7 & 12 \\
SPMS & 0 & 21 & 0 & 6 \\
PPMS & 0 & 0 & 0 & 8 \\
Healthy & 26 & 9 & 10 & 7 \\
\hline & \multicolumn{7}{c}{ (c) Exponential } & \\
RRMS & 19 & 0 & 0 & 0 \\
SPMS & 0 & 3 & 0 & 0 \\
PPMS & 0 & 0 & 5 & 0 \\
Healthy & 0 & 0 & 0 & 6 \\
\hline
\end{tabular}

Table 4 According to Hölder Functions SOM Map Quality for MRI.

\begin{tabular}{lc}
\hline Hölder Function & Ratio Explained \\
\hline Polynomial & $37.25 \%$ \\
Periodic & $88.12 \%$ \\
Exponential & $58.78 \%$ \\
\hline
\end{tabular}

with the best SOM map quality compared to the other two methods.

\section{CONCLUSION}

MRI of 120 individuals with MS subgroup and 19 healthy ones for three different years were worked on. Multifractal has the ability to highlight the significant pixel from image. The $m f B m$ analysis was applied to the 2D pixel, to MRI data. The Hölder function was applied so as to get quality and comprehensive results from the analysis procedure. It was aimed to cluster MS subgroups and healthy individuals based on the SOM algorithm by applying polynomial, periodic and exponential functions to MRI pixels. The Kohonen SOM learning algorithm, one of the unsupervised learning methods was used for the cluster procedure. As a result of the clustering procedure in SOM algorithm, it is possible to show the network structure of all the neurons with their interrelation. Through polynomial, periodic and exponential functions applied to MRI data, $128 \times 128$ important pixels (data obtained from Hölder regularity functions in the
MRI) were selected and provided as inputs neurons to the SOM training algorithm. In our study, the clustering of MS subgroups and healthy subjects were performed by SOM algorithm based on the significant pixel values selected from Hölder regularity functions. The pixel obtained from the periodic Hölder function, with its rate of $88.12 \%$, appeared to be yielding the best cluster analysis result in the SOM training algorithm when compared to the polynomial and exponential functions. This study is aimed to contribute to the filling of a deficiency in the literature through a cluster analysis of MS subgroup and healthy individuals by multifractal pixel processing on MRI data. This study carried out on MS data for the specified three regions of the brain (brainstem, corpus callosum- periventricular region, upper cervical) for the first time in the literature will help physicians in medical practice with the clustering of the MS subgroups by using MRI.

\section{ACKNOWLEDGMENTS}

The authors would like to extend their gratitude to Rana Karabudak and her team as well as to Hacettepe University Medical Faculty Neurology and Radiology Department, Primer Medical Imaging Center for providing medical images. Yeliz Karaca would like to extend her thanks to the distinguished Turkish Neurological Association which granted rewards and financial aids to her. In addition, she is grateful to the Tuscia University, Engineering School (DEIM) for hosting her during this research.

\section{REFERENCES}

1. M. A. Ron, Cognition in multiple sclerosis, Oxford Textbook of Cognitive Neurology and Dementia, A. Husain and J. M. Schott (eds.) (Oxford University Press, Oxford, 2016), p. 295.

2. B. G. Weinshenker, B. Bass, G. P. A. Rice, J. Noseworthy, W. Carriere, J. Baskerville and G. C. Ebers, The natural history of multiple sclerosis: A geographically based study, Brain 112(1) (1989) 133146.

3. C. Solaro and M. M. Uccelli, Pain in multiple sclerosis, An Introduction to Pain and its Relation to Nervous System Disorders (Wiley Blackwell, NJ, 2016), pp. $345-360$.

4. C. Hirst, G. Ingram, R. Swingler, D. A. S. Compston, T. Pickergill and N. P. Robertson, Change in disability in patients with multiple sclerosis: A 20-year prospective population-based analysis, 
J. Neurol. Neurosurg. Psychiatr. Pract. Neurol. 79(10) (2008) 1137-1143.

5. C. Cattani, G. Pierro and G. Altieri, Entropy and multifractality for the myeloma multiple TET 2 gene, Math. Probl. Eng. 2012 (2012) 1-14.

6. L. Trujillo, P. Legrand and J. Lv́y-Vh́el, The estimation of Hölderian regularity using genetic programming, in Proceedings of the 12th Annual Conference on Genetic and Evolutionary Computation, Portland, Oregon, USA, 7-11 July 2010 (ACM, NY, 2010), pp. 861-868.

7. L. Yu and D. Qi, Hölder exponent and multifractal spectrum analysis in the pathological changes recognition of medical CT image, in Chinese Control and Decision Conference 2011 Sichuan Province China, 23-25 May 2011 (IEEE, 2011).

8. M. Phothisonothai and M. Nakagawa, EEG signal classification method based on fractal features and neural network, 30th Annual International Conference of the IEEE Engineering in Medicine and Biology Society (Vancouver, 2008), pp. 3880-3883.

9. J. M. Zook and K. M. Iftekharuddin, Statistical analysis of fractal-based brain tumor detection algorithms, Magn. Reson. Imaging 23(5) (2005) 671678.

10. F. J. Esteban, J. Sepulcre, N. V. de Mendizábal, J. Goñi, J. Navas, J. R. de Miras, B. Bejarano, J. C. Masdeu and P. Villoslada, Fractal dimension and white matter changes in multiple sclerosis, $\mathrm{Neu}$ roimage 36(3) (2007) 543-549.

11. E. Akar, S. Kara, H. Akdemir and A. Kr, Fractal analysis of MR images in patients with chiari malformation: The importance of preprocessing, Biomed. Signal Process. Control. 31 (2017) 63-70.

12. N. Abirami and S. Karthik, Multifractal texture estimation for detection and segmentation of brain tumors with the source and age of the tumor, Int. J. Eng. Sci. 6(3) (2016) 2549-2553.

13. S. Lahmiri and M. Boukadoum, Automatic brain MR images diagnosis based on edge fractal dimension and spectral energy signature, in Annual International Conference of the IEEE Engineering in Medicine and Biology Society (San Diego, 2012), pp. 6243-6246.

14. Y. Karaca, Y. Zhang, C. Cattani and U. Ayan, The differential diagnosis of multiple sclerosis using convex combination of infinite kernels, CNS Neurol. Disord. Drug Targets 16(1) (2016) 36-43.

15. D. M. Sima, D. Loeckx, D. Smeets, S. Jain, P. M. Parizel and W. Van Hecke, Focus on multiple sclerosis, in Imaging Biomarkers Use Case I: Imaging Biomarkers in Neurological Disease (Springer, Switzerland, 2017), pp. 169-180.
16. R. F. Peltier and J. Lévy-Véhel, Multifractional Brownian motion: Definition and preliminary results, Research Report, NRIA, France (1955).

17. B. Pesquet-Popescu and J. L. Véhel, Stochastic fractal models for image processing, IEEE Signal Process. Mag. 19(5) (2002) 48-62.

18. T. T. Marquez-Lago, A. Leier and K. Burrage, Anomalous diffusion and multifractional Brownian motion: Simulating molecular crowding and physical obstacles in systems biology, IET Syst. Biol. 6(4) (2012) 134-142.

19. G. Chan and A. T. A. Wood, Simulation of multifractional Brownian motion: in COMPSTAT, Proceedings Computational Statistics, 13th Symposium (Bristol, UK, 1998), pp. 233-238.

20. A. Ayache and J. L. Véhel, On the identification of the pointwise Hölder exponent of the generalized multifractional Brownian motion, Stoch. Process. Appl. 111(1) (2004) 119-156.

21. B. Pesquet-Popescu and J. Lévy-Véhel, Stochastic fractal models for image processing, IEEE Signal Process 19(5) (2002) 48-62.

22. J. Lévy-Véhel, Fractal approaches in signal processing, Fractals 3 (1995) 755-775.

23. M. Fhima, A. Guillin and P. R. Bertrand, Fast change point analysis on the Hurst index of piecewise fractional Brownian motion, in Proceedings: Journee de Statistiques (Tunisia, 2011), 416 p.

24. A. K. B. Chand, M. A. Navascues, P. Viswanathan and S. K. Katiyar, Fractal trigonometric polynomials for restricted range approximation, Fractals 24(02) (2016) 1-11.

25. B. B. Mandelbrot and J. W. Van Ness, Fractional Brownian motions, fractional noises and applications, SIAM Rev. 10(4) (1968) 422-437.

26. G. Chan and A. T. Wood, Simulation of multifractional Brownian motion, in Proceeding COMPSTAT (Physica-Verlag HD, 1998), pp. 233-238.

27. R. F. Peltier and J. L. Vhel, Multifractional Brownian motion: Definition and preliminary results Doctoral dissertation, INRIA (1995).

28. B. H. Gilding, Hölder continuity of solutions of parabolic equations, J. Lond. Math. Soc. 2(1) (1976) $103-106$.

29. L. Trujillo, P. Legrand, G. Olague and J. LevyVehel, Evolving estimators of the pointwise Hölder exponent with genetic programming, Inf. Sci. 209 (2012) 61-79.

30. T. Kohonen, Self-Organization and Associative Memory, Vol. 8 (Springer Science \& Business Media, NY, 2012).

31. D. I. Kumar and M. R. Kounte, Comparative study of self-organizing map and deep self-organizing map 
using MATLAB 2016, in Proceedings in Communication and Signal Processing, International Conference IEEE Chennai, India, 6-8 April 2017, pp. 1020-1023.

32. S. Haroun, A. N. Seghir and S. Touati, Feature selection for enhancement of bearing fault detection and diagnosis based on self-organizing map, in Recent Advances in Electrical Engineering and Control Applications (Springer, Switzerland, 2017), pp. 233-246.

33. T. Kohonen, MATLAB Implementations and Applications of the Self-Organizing Map (Unigrafia, Helsinki, 2014), pp. 21-26. 